\section{(2) OPEN ACCESS}

\title{
Impairment of spermatogenesis and sperm motility by the high-fat diet-induced dysbiosis of gut microbes
}

\author{
Ning Ding $\odot 1,{ }^{1}$ Xin Zhang, ${ }^{2}$ Xue Di Zhang, ${ }^{1}$ Jun Jing, ${ }^{3,4}$ Shan Shan Liu, ${ }^{5}$ Yun Ping Mu, \\ Li Li Peng (1) , ${ }^{6}$ Yun Jing Yan, ${ }^{1}$ Geng Miao Xiao, ${ }^{1}$ Xin Yun Bi, ${ }^{1}$ Hao Chen, ${ }^{1}$ \\ Fang Hong Li, ${ }^{1}$ Bing Yao, ${ }^{3,4}$ Allan Z Zhao ${ }^{1}$
}

- Additional material is published online only. To view, please visit the journal online (http://dx.doi.org/10.1136/ gutjnl-2019-319127).

The School of Biomedical and Pharmaceutical Sciences, Guangdong University of Technology, Guangzhou, Guangdong, China

2Dizal Pharma, Shanghai, China ${ }^{3}$ Jinling Hospital Department Reproductive Medical Center, Nanjing Medicine University, Nanjing, Jiangsu, China

${ }^{4}$ State Key Laboratory of Reproductive Medicine, Nanjing Medical University, Nanjing, Jiangsu, China

${ }^{5}$ Department of Laboratory, Women and Children 's Hospital of Qingdao, Qingdao, Shandong, China

${ }^{6}$ The School of Biology and Biological Engineering, South China University of Technology, Guangzhou, Guangdong, China

\section{Correspondence to}

Dr Allan Z Zhao, The School of Biomedical and Pharmaceutical Sciences, Guangdong University of Technology, Guangzhou, China; azzhao@gdut.edu.cn Dr Bing Yao; yaobing@nju.edu.cn Dr Fang Hong Li; fli@gdut.edu.cn

Dr Hao Chen;

chenhao@gdut.edu.cn

Received 19 May 2019 Revised 9 December 2019 Accepted 10 December 2019 Published Online First 2 January 2020

\section{Check for updates}

(C) Author(s) (or their employer(s)) 2020. Re-use permitted under CC BY-NC. No commercial re-use. See rights and permissions. Published by BMJ.

To cite: Ding N, Zhang $X$, Zhang XD, et al. Gut

2020;69:1608-1619.

\section{ABSTRACT \\ Objective High-fat diet (HFD)-induced metabolic disorders can lead to impaired sperm production. We aim to investigate if HFD-induced gut microbiota dysbiosis can functionally influence spermatogenesis and sperm motility.}

Design Faecal microbes derived from the HFD-fed or normal diet (ND)-fed male mice were transplanted to the mice maintained on ND. The gut microbes, sperm count and motility were analysed. Human faecal/semen/blood samples were collected to assess microbiota, sperm quality and endotoxin.

Results Transplantation of the HFD gut microbes into the ND-maintained (HFD-FMT) mice resulted in a significant decrease in spermatogenesis and sperm motility, whereas similar transplantation with the microbes from the ND-fed mice failed to do so. Analysis of the microbiota showed a profound increase in genus Bacteroides and Prevotella, both of which likely contributed to the metabolic endotoxaemia in the HFD-FMT mice. Interestingly, the gut microbes from clinical subjects revealed a strong negative correlation between the abundance of Bacteroides-Prevotella and sperm motility, and a positive correlation between blood endotoxin and Bacteroides abundance. Transplantation with HFD microbes also led to intestinal infiltration of $T$ cells and macrophages as well as a significant increase of pro-inflammatory cytokines in the epididymis, suggesting that epididymal inflammation have likely contributed to the impairment of sperm motility. RNA-sequencing revealed significant reduction in the expression of those genes involved in gamete meiosis and testicular mitochondrial functions in the HFD-FMT mice.

Conclusion We revealed an intimate linkage between HFD-induced microbiota dysbiosis and defect in spermatogenesis with elevated endotoxin, dysregulation of testicular gene expression and localised epididymal inflammation as the potential causes.

Trial registration number NCT03634644.

\section{INTRODUCTION}

Infertility is a global issue that affects as much as $10 \%-15 \%$ of couples in many high-income countries and in countries with improving dietary conditions. ${ }^{1}$ Approximately half of the infertility can be attributed to men. Of note, the sperm concentration has decreased over $50 \%$ in Western countries from 1973 to 2011 and there is still no effective

\section{Significance of this study}

What is already known on this subject?

- High-fat diet (HFD) leads to obesity and metabolic syndromes and affects the functions in reproductive system.

- HFD-induced gut microbiota dysbiosis and endotoxaemia have been reported.

- Emerging evidence demonstrated that gut microbiota dysbiosis is closely associated with a series of major chronic diseases, such as obesity, diabetes, cancer, IBDs and neurodegenerative diseases.

treatment to slow down such a trend. ${ }^{2}$ The rapid decline of semen quality is likely due to the environmental factors rather than genetic causes, ${ }^{34}$ such as environmental toxin, heat, stress, ${ }^{5}$ smoking, ${ }^{6}$ alcohol consumption ${ }^{7}$ and altered dietary structures. Furthermore, with the prevalence of obesity, the negative impact of fat overaccumulation on male reproduction is gradually recognised in clinical investigations. ${ }^{8-11}$ In particular, a recent study involving >10 000 subjects clearly demonstrated that the sperm quality, including sperm concentration, volume and motility, is negatively correlated with increased body mass index. ${ }^{12}$ In animal models, both genetically inherited and high-fat diet (HFD)induced obesity impaired spermatogenesis. ${ }^{13}{ }^{14}$ Furthermore, HFD could also lead to epigenetic disturbance $^{15}$ in sperms as paternal inheritance of sperm tsRNAs could induce metabolic disorders in the F1 offspring. ${ }^{16}$

In both humans and animals, the high-fat/highsugar 'Western' diet can lead to obesity and metabolic disorders and reshape the gut microbial ecosystem ${ }^{17}{ }^{18}$ comprising trillions of microorganisms. ${ }^{19}$ These microbes in the mammal intestine modulate many physiological functions, such as harvesting energy, ${ }^{20}$ keeping gut integrity, ${ }^{21}$ regulating host immunity 22 and defending pathogens. $^{2425}$ The perturbation of the 'healthy' gut microbiota may drive the development of a variety of chronic diseases, such as obesity, ${ }^{20}{ }^{26}$ cancer, ${ }^{22}$ IBDs $^{27}$ and diabetes. ${ }^{28}$ However, it is still unknown if HFD can impair reproduction through gut microbiota. Herein, we designed a study to delineate the 
Significance of this study

What are the new findings?

- Transplantation of the faecal microbes derived from HFDfed mice into the male mice maintained on normal diet resulted in significant reduction in sperm count and motility, which represents the first report of functional impact of gut microbes dysbiosis on fertility.

- After extensive bioinformatics analysis, we showed that, at the genus level, the population of two Gram-negative bacteria, Bacteroides and Prevotella, was sharply elevated in the gut transplanted with HFD-microbe.

- Concomitantly, blood endotoxin reached the level of endotoxaemia in the same transplanted mice.

- Clinically, the abundance of Bacteroides-Prevotella was negatively correlated with subjects' sperm motility, the blood endotoxin level was positively correlated with the abundance of Bacteroides in the gut.

- A strong negative correlation was found between sperm motility and high abundance of Prevotella copri (>15\% in overall species).

- Transplantation and colonisation of the microbes derived from HFD-fed mice caused significant increase in intestinal infiltration of $\mathrm{CD}^{+} \mathrm{T}$ cells and macrophages as well as in epididymal pro-inflammatory cytokines, suggesting that endotoxin and epididymal inflammation play important roles in gut microbe dysbiosis-induced disruption of spermatogenesis.

How might it impact on clinical practice in the foreseeable future?

- The present study represents the first report in the field of gut microbes to functionally establish the linkage between imbalanced gut microbiota and impairment of male fertility, and that some of these gut microbe dysbiosis has also been revealed in asthenozoospermia patients.

- The results presented herein will shed new light on the cause of male infertility and the possibility of treating at least some of these patients by restoring gut microbial ecosystem.

impact of HFD-induced gut microbial dysbiosis on the production and motility of sperms.

\section{MATERIALS AND METHODS}

Collection of human semen, plasma and faecal samples

Human semen, plasma and faecal samples were obtained from donors aged 22-45years undergoing routine semen analysis for healthy or infertile patients in our clinical laboratory (online supplementary table 1). Sperm quality was assessed by a computer-assisted semen analysis (CASA, WLJY-9000) system according to WHO suggestions. ${ }^{29}$ Normal sperm criteria defined as sperm concentration $>15 \times 10^{6} / \mathrm{mL}$, progressive motility $>32 \%$, total motility $>40 \%$ and normal sperm morphology rate $>4 \%$. Progressive motility $<32 \%$ and total motility $<40 \%$ is defined as asthenozoospermia.

\section{Animal procedures}

C57BL/6 male mice aged 6 weeks were purchased from the Model Animal Research Center of Nanjing University, and maintained in specific pathogen-free (SPF) environment on a 12-hour light and dark cycle with ad libitum access to chow and water. Mice were randomly divided into normal diet (ND),
HFD, normal-diet faecal microbiota transplantation (ND-FMT) and high-fat diet faecal microbiota transplantation (HFD-FMT) groups (six mice per group, three mice per cage). The ND group and the HFD group were fed with an ND or an HFD (Research Diet D12451, 45\% fat), respectively. Following 4 weeks of dietary intervention, the faecal samples collected from either the ND or HFD mice were subsequently transplanted into the ND-FMT or HFD-FMT group that were maintained on an ND (see 'Fecal microbiota transplantation experiment'). All mice were sacrificed after 15 weeks of either dietary or FMT intervention for the harvest of serum and tissues.

\section{Faecal microbiota transplantation experiment}

Two hundredmg of fresh stool samples were collected from the ND and HFD group immediately on defaecation and resuspended in $4 \mathrm{~mL}$ of saline, vortexed for $5 \mathrm{~min}$ and filtered by sterile gauze. The transplantation into the recipient mice was achieved by gavage with $200 \mu \mathrm{L}$ of the supernatant from the faecal sample once every 2 days for 15 weeks.

\section{Sperm count and motility measurement}

The cauda epididymis was minced in $500 \mu \mathrm{L}$ of phosphate buffered saline ( $\mathrm{pH} \mathrm{7.2)}$ and incubated for $5 \mathrm{~min}$ at $37^{\circ} \mathrm{C}$ to release the sperm. Sperm motility was measured with a (Hamilton) equipment. The sperm count was quantified in a haemocytometer (Marienfeld AP-0650030) under a light microscope (Leica DM500).

\section{Histological examination}

Testes or intestines were removed and fixed in 4\% formalin for 24 hours, dehydrated in graded ethanol, embedded in paraffin and sectioned ( $4 \mu \mathrm{m}$ in thicknesses). The sections were stained with H\&E dye and observed under light microscope (Leica DM500). Six microscopic fields of every section of the testes were randomly selected $(\times 400 \text { magnification })^{30}$ and different germ cells (spermatogonia, spermatocytes and round spermatids) were counted ( $n=6 /$ group). Data are presented as mean \pm SEM.

\section{Confocal microscopy and immunofluorescence}

For immunofluorescence staining, paraffin sections $(4 \mu \mathrm{m}$ thicknesses) were assayed with 4',6-diamidino-2-phenylindole (DAPI) as a counterstain. The primary antibody used was rabbit antimouse CD3e (1:400; Abcam). The secondary antibody (1:500; Invitrogen) used was Alexa Fluor488 donkey antirabbit. Sections were examined under a Zeiss LSM800 confocal microscope.

\section{Immunohistochemical staining}

The paraffin tissue sections ( $4 \mu \mathrm{m}$ thicknesses) were incubated with antibody against F4/80 (1:400; Cell Signaling Technology). The specific signals were detected using EnVision polymer technology, and visualisation was performed with 3,3N-diaminobenzidine tetrahydrochloride. The sections were photographed by LEICA DM2500 microscope with a LEICA DMC6200 camera.

\section{Measurement of serum parameters}

Mouse serum endotoxin was measured by ELISA kits (Nanjing Jiancheng Bioengineering Institute, China) in accordance with the manufacturer's protocols. Human plasma endotoxin was measured by end-point chromogenic TAL endotoxin testing assay (Bioendo, China). 


\section{Oral glucose tolerance test}

After 12 hours of fasting, all mice underwent an oral glucose tolerance test. Following oral gavage of D-glucose $(2 \mathrm{~g} / \mathrm{kg}$ body weight), blood samples were collected from the tail veins at 0 , 15, 30, 45, 60, 90 and $120 \mathrm{~min}$. Glucose levels were determined with a glucometer (Accu-Chek Performa).

\section{Extraction of testis RNA and sequencing}

Total RNA was extracted from testis tissues using TRIzol reagent kit (Invitrogen, Carlsbad, California, USA). Subsequently, the mRNA was enriched by oligo (dT) beads. The enriched mRNA was fragmented with fragmentation buffer and reversely transcribed into cDNA with random primers. Second-strand cDNA were synthesised by DNA polymerase I, RNase $\mathrm{H}$, dNTP and buffer. The cDNA fragments were purified with a QiaQuick PCR extraction kit (Qiagen 28104), end repaired, poly(A) added and ligated to Illumina sequencing adapters. The ligation products were size selected by agarose gel electrophoresis, PCR amplified and sequenced using Illumina HiSeq 4000 by Gene Denovo Biotechnology (Guangzhou, China). To identify differentially expressed genes across groups, the edgeR package (http://www. r-project.org/) was used. We identified genes with a fold change $\geq 2$, a false discovery rate $<0.05$ and Fragments Per Kilobase of exon per million fragments Mapped (FPKM) $\geq 3$ in a comparison as significant differentially expressed genes. Raw reads were deposited into the NCBI Sequence Read Archive (SRA) database. Accession number: SRP167405.

\section{Real-time PCR}

Total RNA was reversely transcribed and the subsequent cDNA samples were subjected to real-time PCR using SYBR Green (Vazyme, China) and the primer sets. Primer sequences for the targeted mouse genes are listed in the online supplementary table 2. Data were normalised to $\beta$-actin $\left(\Delta \Delta \mathrm{C}_{\mathrm{T}}\right.$ analysis).

\section{Extraction of faecal genomic DNA and sequencing}

Faecal DNA was extracted from caecum stool samples using a QIAamp DNA Stool Mini Kit (Qiagen 51504) according to manufacturer's instructions. The hypervariable V3-V4 region of the 16S-rDNA gene was amplified by PCR using the primers 341F: CCTACGGGNGGCWGCAG; 806R: GGACTACHVGGGTATCTAAT, where the barcode was an eight-base sequence unique to each sample. Amplicons were recovered from 2\% agarose gels and purified using the AxyPrep DNA Gel Extraction Kit (Axygen Biosciences, USA) according to manufacturer's instructions and quantified using a QuantiFluor-ST (Promega, USA). The purified amplicons were pooled in equimolar and paired-end sequenced $(2 \times 250)$ on an Illumina platform according to the standard protocols. The raw reads were deposited into the NCBI SRA database with accession number: SRP168312 for mouse faecal samples; SRP221703 for human samples.

\section{Bioinformatics and statistical analysis of 16S-rDNA sequencing data}

To get high-quality clean reads, the standard for selected valid reads for analysis were as follows: (a) removing reads containing $>10 \%$ of unknown nucleotides $(\mathrm{N})$, (b) removing reads containing $<80 \%$ of bases with quality (Q-value) $>20$. Paired end clean reads were merged as raw tags using FLSAH (V.1.2.11) with a minimum overlap of $10 \mathrm{bp}$ and mismatch error rates of $2 \%$. Noisy sequences of raw tags were filtered by QIIME (V.1.9.1) pipeline under specific filtering conditions to obtain the high-quality clean tags. The effective tags were clustered into operational taxonomic units (OTUs) of $\geq 97 \%$ similarity using MOTHUR pipeline. The tag sequence with highest abundance was selected as reprehensive sequence within each cluster. The representative sequences were classified into organisms by a naïve Bayesian model using RDP classifier (V.2.2) based on Greengenes (V.gg_13_5) (http://greengenes.secondgenome. com). The abundance statistics of each taxonomy and phylogenetic tree was construction in a Perl script and visualised using Scalable Vector Graphics. Principal coordinate analysis (PCoA) of weighted unifrac distances was calculated and plotted in R.

\section{Statistical analysis}

Data are expressed as mean \pm SEM. Statistical comparison between two measurements were analysed by unpaired twotailed Student's t-test by GraphPad Prism V.7.0. When analysing gut microbiota sequencing data, we performed two-tailed Wilcoxon rank-sum test by R Project; $\mathrm{p}<0.05$ was considered to be statistically significant.

\section{RESULTS \\ High-fat diet results in decreased sperm quality and dysbiosis of gut microbiota}

To study the influence of HFD-induced dysbiosis on the spermatogenesis and sperm motility, we first set out to establish a HFD-fed (45\% fat) mouse model and a control model fed the ND to serve as the faecal microbiota donors. As one would expect, the extended HFD regimen led to increased body weight (online supplementary figure 1A), elevated serum total cholesterol (TC), random blood glucose levels (online supplementary figure $1 \mathrm{~B}$ ) and glucose intolerance (online supplementary figure 1C), suggesting that HFD induced metabolic disorders. Also consistent with the findings in the previous reports, ${ }^{1826}$ the HFD caused gut microbiota dysbiosis as revealed in the 16S-rDNA sequencing analysis. Accordingly, a weighted PCoA clearly showed differences in bacterial composition between the HFD and ND groups (figure 1A) with a profound difference along the PCO1 axis (reaching 63.13\% of overall variation). In addition, HFD treatment also led to a decreased abundance in Bacteriodetes and Verrucomicrobia as well as an increased abundance in Firmicutes and Proteobacteria (figure 1B). Of particular interest to this study, the sperm concentration (figure 1C) and motility (figure 1D) were significantly decreased in the HFD-treated mice. H\&E staining analysis further indicated significant reduction in the number of spermatocytes and round spermatids in the seminiferous tubules (figure $1 \mathrm{E}$, online supplementary table $3)$. These observations were in line with those reported elsewhere. ${ }^{81431}$ Thus, the HFD treatment induced overt changes in metabolic parameters and the expected gut microbial dysbiosis and sperm quality.

\section{Impaired spermatogenesis and sperm motility in the HFD-FMT mice}

To investigate the impact of the HFD-induced microbiota dysbiosis on sperm concentration and motility, we transferred the faecal samples from the HFD donors or ND donors to the C57BL/6 male mice maintained on the ND. Since some recent studies revealed that antibiotics increase host susceptibility to obesity ${ }^{32}$ in humans and that pretreatment with antibiotics before FMT could lead to glucose intolerance, ${ }^{33}$ these entangling metabolic side effects may cause spermatogenic defects. Furthermore, all taxa analysis of the faecal bacterial DNA samples derived from eight randomly selected recipient male mice did 
A

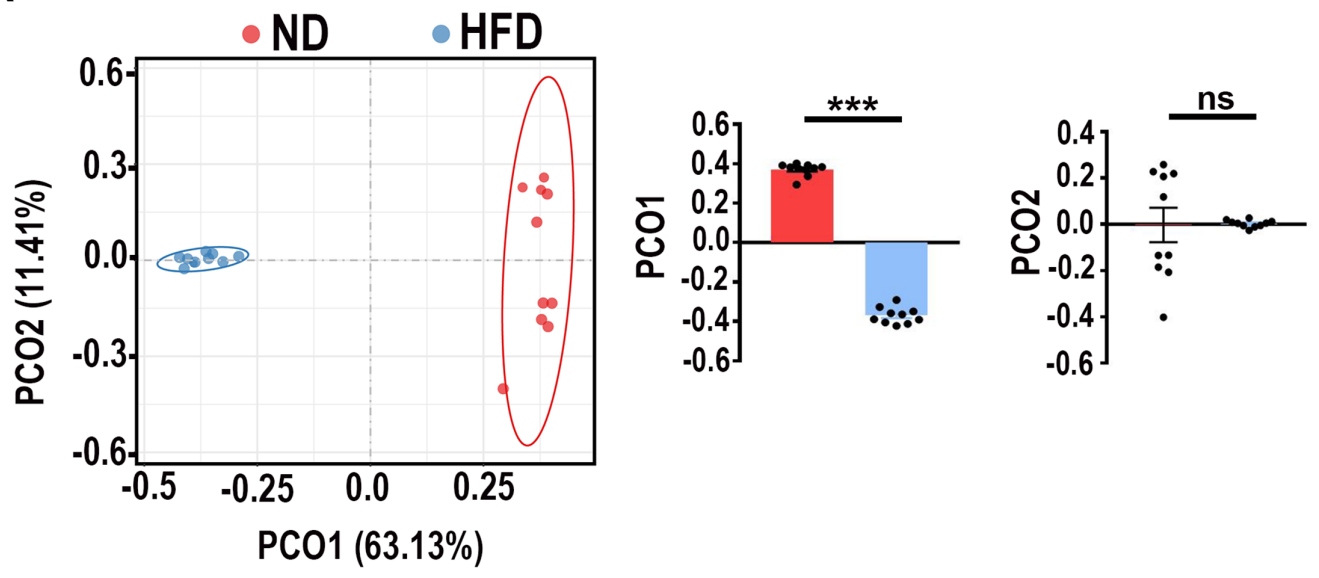

B

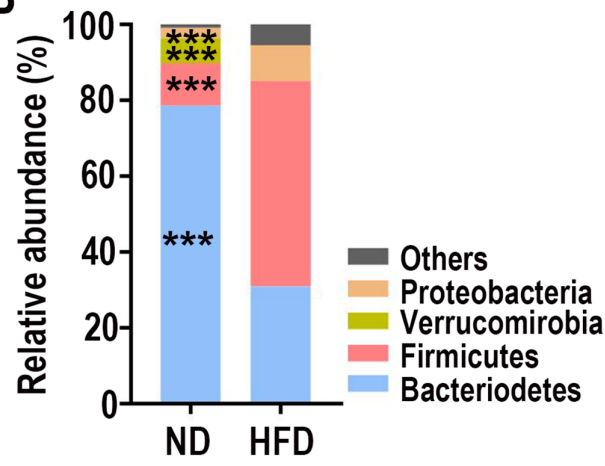

C

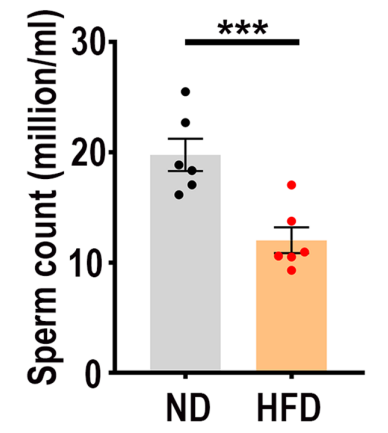

D

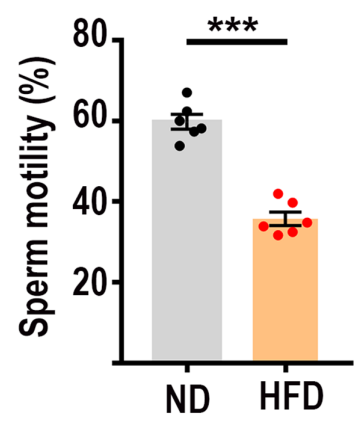

E
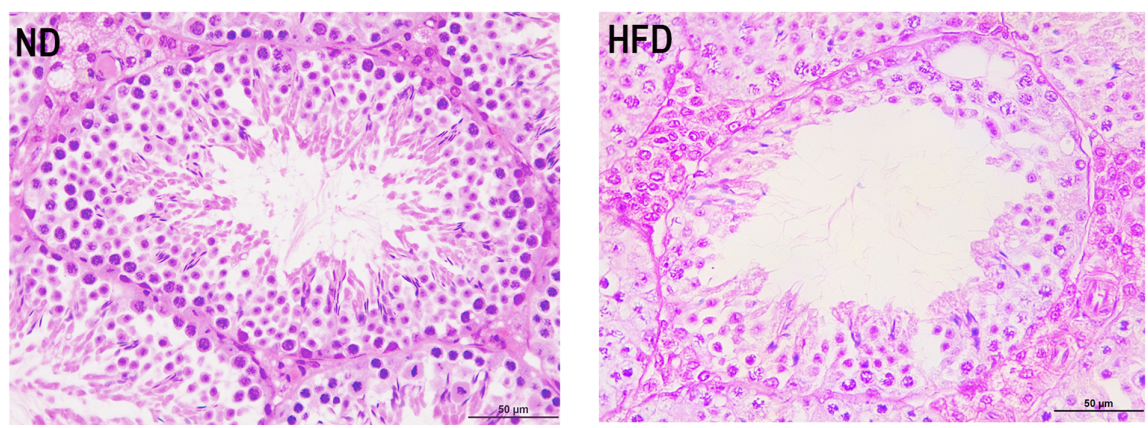

Figure 1 High-fat diet (HFD) altered gut microbiota with impaired sperm production and motility. (A) Principal coordinate analysis (PCoA) plot is generated using operational taxonomic unit metrics based on the Bray-Curtis similarity for the samples in the normal diet (ND) and HFD groups. The centre point coordinate of the ellipse is the mean value of PCO1 and PCO2, respectively, in the corresponding group. The values of PCO1 and PCO2 are shown in bar plots, $n=10$ for each group. (B) The mean percentages of each community contributed by the indicated phyla, $n=10$ for each group. (C) Sperm from cauda epididymis of mice in the ND group or the HFD group was counted in a haemocytometer under a light microscope, $\mathrm{n}=6$ for each group. (D) Sperm motility of mice in the ND group and the HFD group was analysed by computer-assisted semen analysis, $n=6$ for each group. Data are expressed as mean $\pm S E M$. ${ }^{* *} p<0.001$. (E) Histological examination in the HFD-treated testis. The sections were stained with H\&E. ns, no significant difference. Scale bar $=50 \mu \mathrm{m}$.

not show any discernible difference (online supplementary table 4). Therefore, we chose to directly transfer the faecal microbial samples without antibiotic pretreatment for the recipient mice. The FMT procedures were carried out once every 2 days for 15 weeks (figure 2A). At the end of this microbial transplantation regimen, the sperm concentration and motility were significantly reduced in the HFD-FMT mice (figure $2 \mathrm{~B}, \mathrm{C}$ ), which was accompanied by a marked decrease of spermatogenic cells within seminiferous tubules (figure 2D). To further dissect the population changes of different cell types, we used the expression levels of
Hsd3b1, Rhox5, Sohlh1, Smc3, Izumo3 and Acrv1 to reflect the alterations of Leydig cell, Sertoli cell, spermatogonia, spermatocytes, elongated spermatids and round spermatids, respectively. ${ }^{34}$ The results showed that the expression of Smc3, Izumo3 and Acrv1 was significantly decreased in the HFD-FMT testicular samples compared with those in the ND-FMT mice, and that the expression levels of Hsd3 $\beta 1$, Rhox 5 and Sohlh1 were essentially the same (figure 2E), indicating that only spermatocytes and spermatids were negatively affected due to the HFD-FMT manipulation. During the entire faecal microbial transfer period, 
A

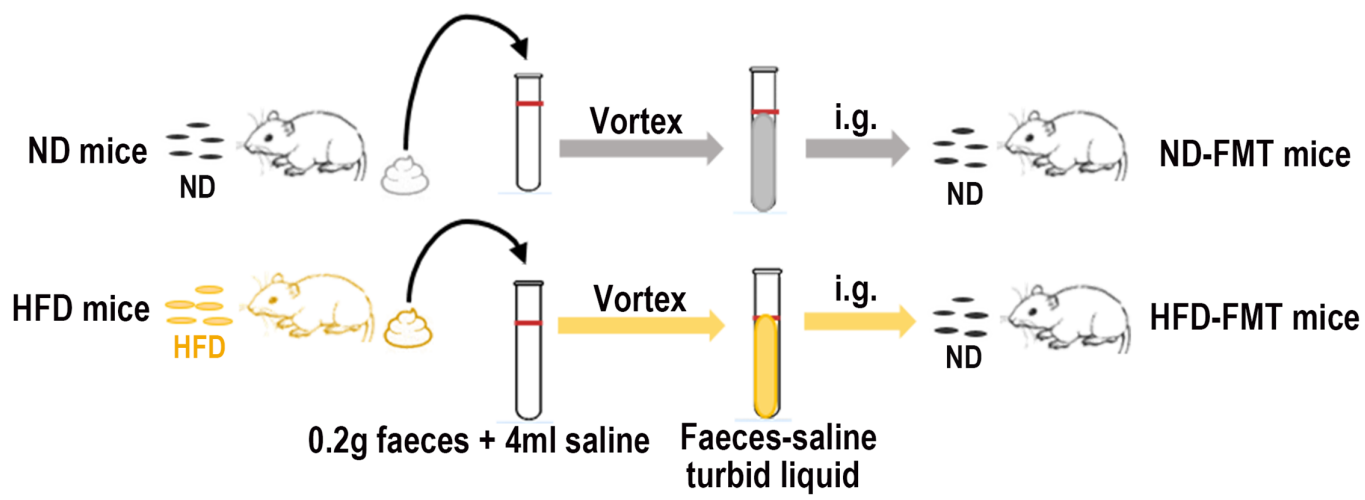

B

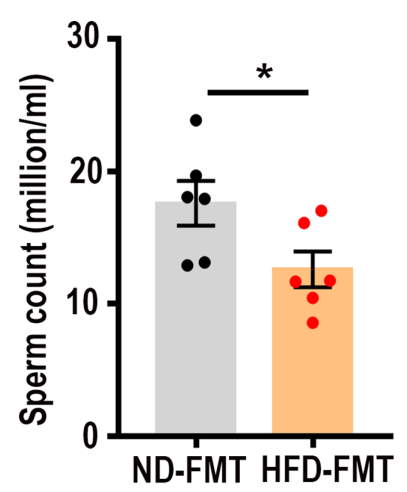

C

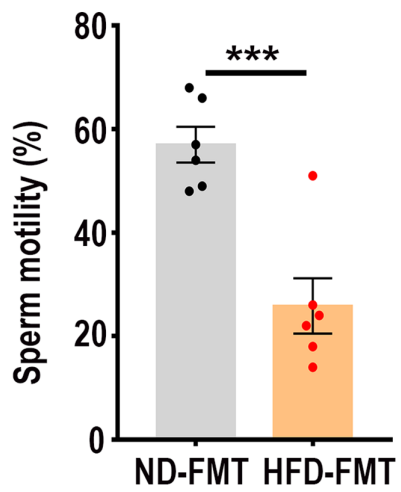

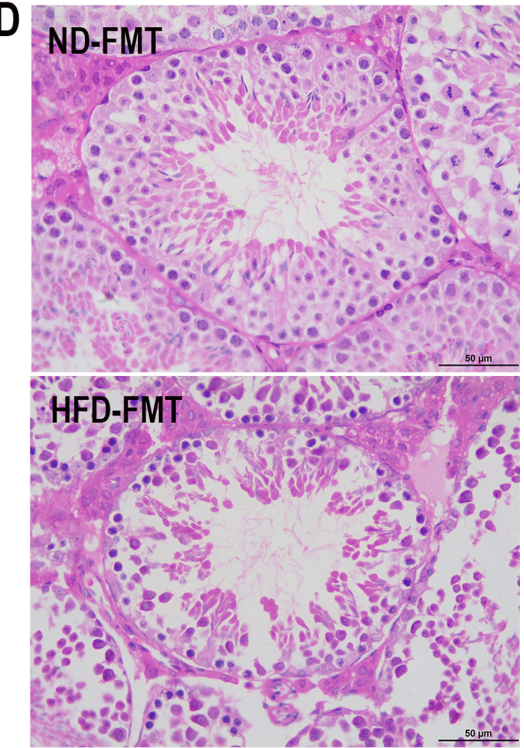

$\mathbf{F}$

E

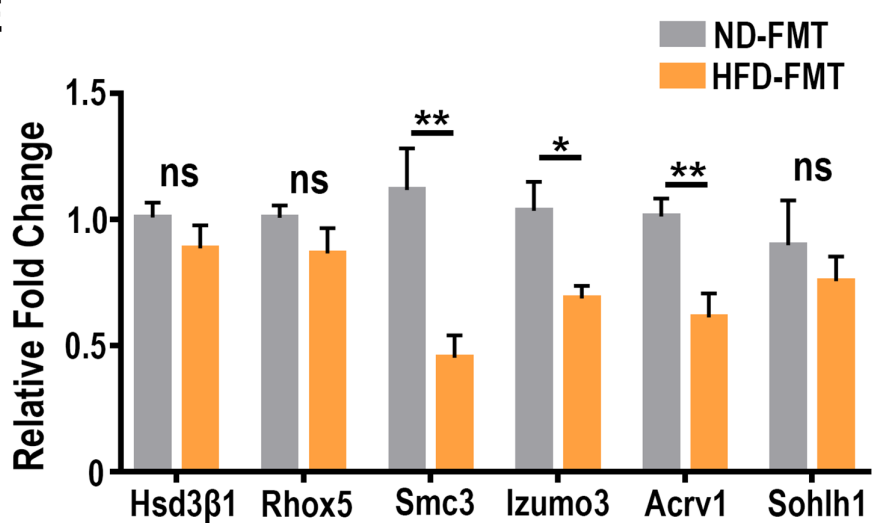

Figure 2 Faecal microbiota transplanted from the HFD mice reduced sperm quality in recipient mice. (A) Schematic diagram of faecal microbiota transplantation experiment. ND, normal diet; HFD, high-fat diet; ND-FMT, normal diet faecal microbiota transplantation; HFD-FMT, high-fat diet faecal microbiota transplantation; i.g., intragastric gavage. (B) Statistical analysis of sperm counts after FMT. Sperm from cauda epididymis of mice in the ND-FMT group or the HFD-FMT group was counted in a haemocytometer under a light microscope. (C) Motility of sperm from the ND-FMT and the HFD-FMT mice was assessed by computer-assisted semen analysis. (D) Representative H\&E staining images showing the impairment of seminiferous tubule in the HFD-FMT testis. Scale bar $=50 \mu \mathrm{m}$. (E) The expression levels of the genes associated with different testicular cells were determined by real-time PCR. (F) Serum endotoxin levels in the ND-FMT mice and the HFD-FMT mice, $n=6$ for each group. ns, no significant difference. ${ }^{*} p<0.05$, ${ }^{* *} \mathrm{p}<0.01,{ }^{* * *} \mathrm{p}<0.001$.

the body weight (online supplementary figure $2 \mathrm{~A}$ ), food intake (online supplementary figure $2 \mathrm{~B}$ ), energy expenditure (online supplementary figure 2C), blood glucose levels, TC levels (online supplementary figure 2D) and circulating leptin levels (online supplementary figure 2E) displayed no ostensible changes. In contrast, the blood endotoxin levels were significantly elevated, even reaching the level of endotoxaemia (figure $2 \mathrm{~F}$ ). Taken together, these findings indicate that HFD-induced dysbiosis of 
A

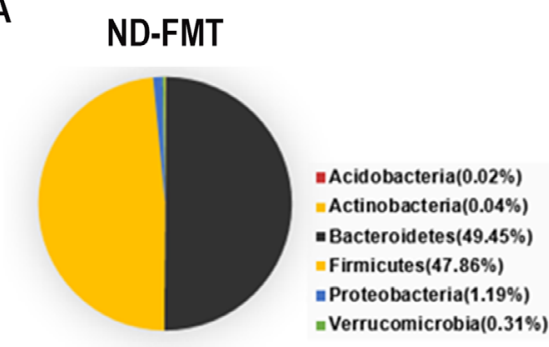

B

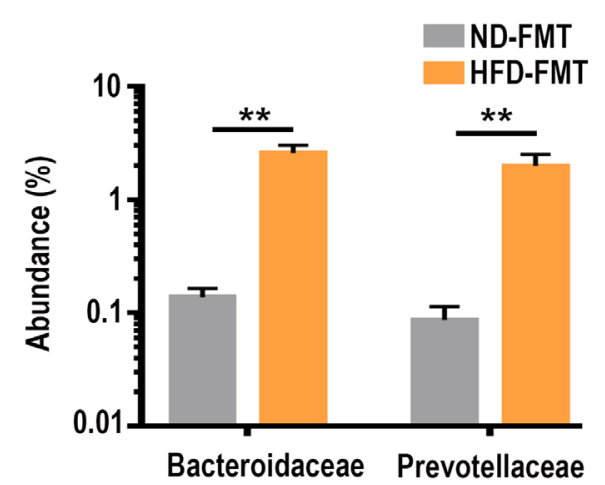

HFD-FMT

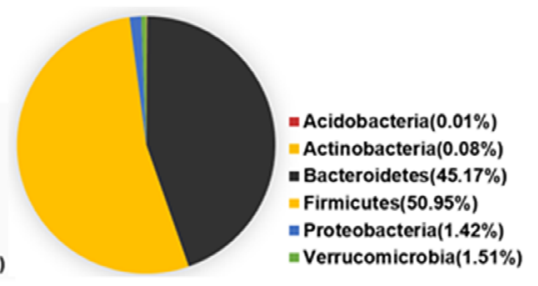

C

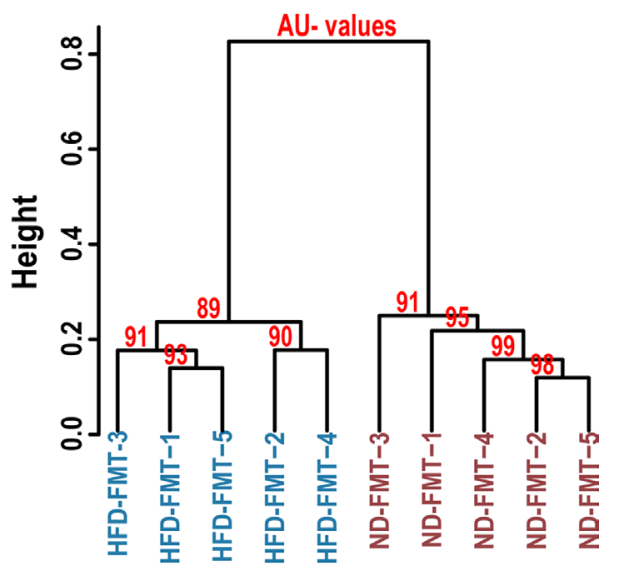

ND-FMT

HFD-FMT

D

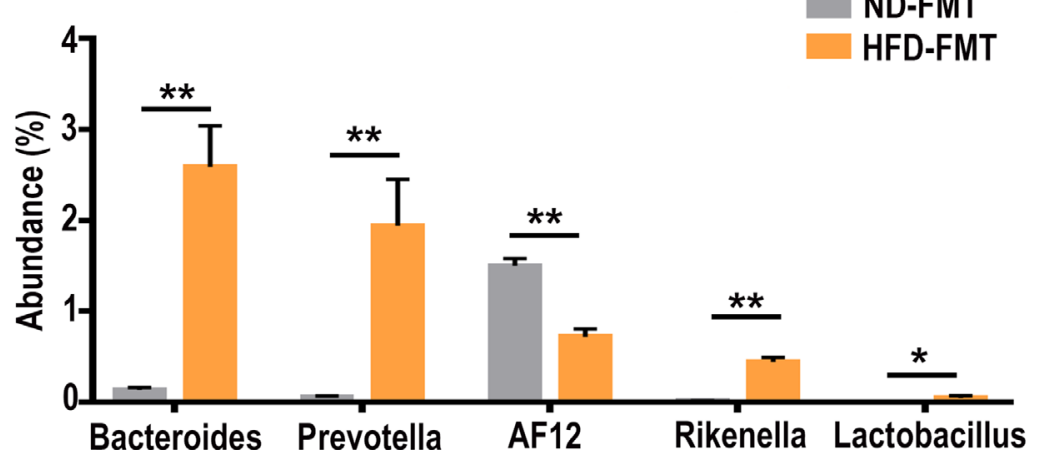

Figure 3 Diet-dependent and FMT-responsive alteration of gut microbiota community. (A) Phylum comparison for the abundance of gut microbiota between the ND-FMT and HFD-FMT treated mice. No significant difference in the microbe composition between the two groups. (B) Abundance of family microbes in HFD-FMT mice was compared with the ND-FMT mice. Two-tailed Wilcoxon rank-sum test was used to determine significance. The average abundance of microbes $>0.01 \%$ was compared. ${ }^{*} p<0.01$. (C) Similarity analysis of microbes on the genus level was compared in the NDFMT and the HFD-FMT treated mice. AU values, approximately unbiased values. (D) Abundance of genera microbes in HFD-FMT mice was compared with the ND-FMT mice, $n=5$ for each group. The average abundance of microbes $>0.01 \%$ was compared. ${ }^{*} p<0.05,{ }^{*} p<0.01$.

gut microbiota could result in male infertility without inducing significant metabolic dysregulations.

\section{The dysbiosis of gut microbiota after faecal microbiota transplantation}

Sequencing of caecum bacterial 16S-rDNA revealed gut microbial ecosystem after the mice were subjected to 15 weeks of transplantation with the faecal bacteria derived from either the HFD-fed or ND-fed mice. Since all recipient mice were maintained on ND, the primary population of gut microbiota at the phylum level, particularly Bacteroidetes, Firmicutes, Proteobacteria and Verrucomicrobia, were essentially the same in the HFD-FMT and ND-FMT mice (figure 3A), and these two groups did not show separate clusters at the phylum level (online supplementary figure 3). At either the class or the order level, there was no significant change in the predominant forms $(>0.5 \%)$ of bacteria (online supplementary table 5). Further bioinformatics analysis along the taxonomic tree revealed some significant changes at the family level, specifically in Bacteroidaceae and Prevotellaceae (figure 3B). Of particular interest to this study, the strong increase in Bacteroidaceae mirrored the increase in the HFD-treated group (online supplementary figure 4 left). Further down at the genus level, the HFD-FMT and ND-FMT groups became clustered to different nodes, and exhibited structural segregation (figure 3C). The population analysis found a profound increase in Bacteroides, Prevotella, Rikenella and Lactobacillus, and a decrease in the abundance of AF12 in the HFD-FMT group (figure 3D). The drastic elevation ( $>80$-fold) of Bacteroides, belonging to the Bacteroidaceae family, was also significantly induced in the original HFD-treated group (online supplementary figure 4 right). 
A

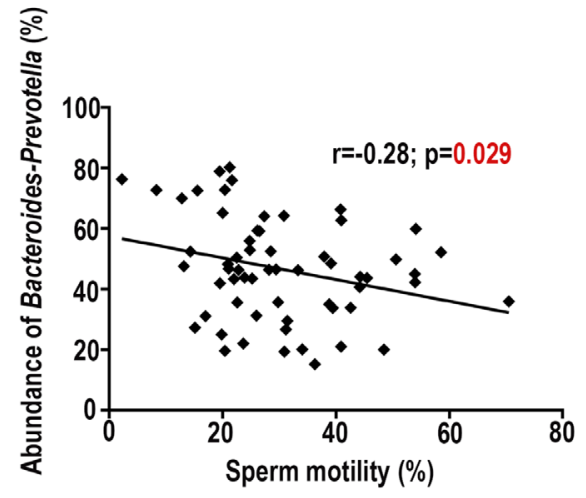

C

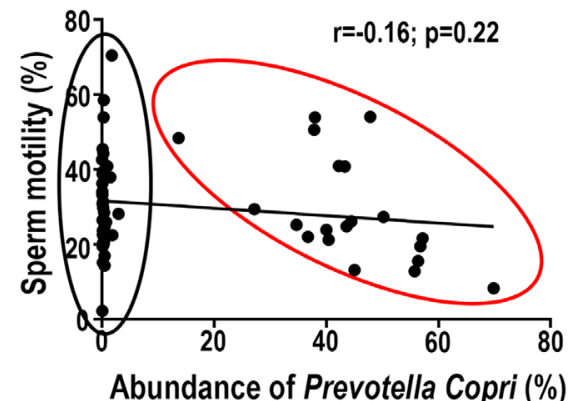

B

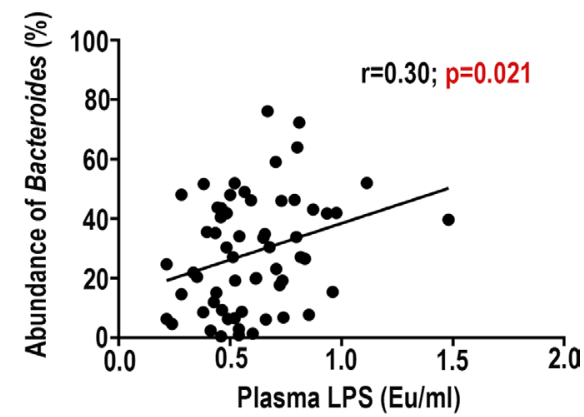

D

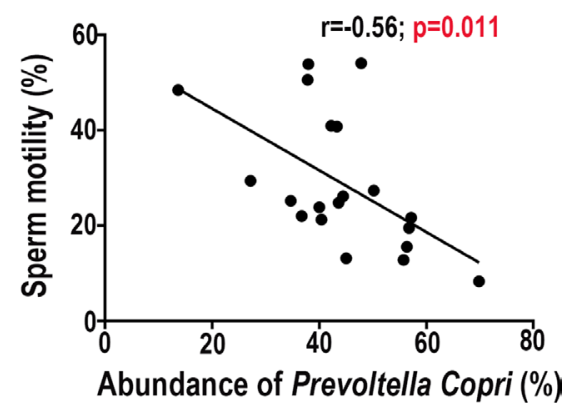

E

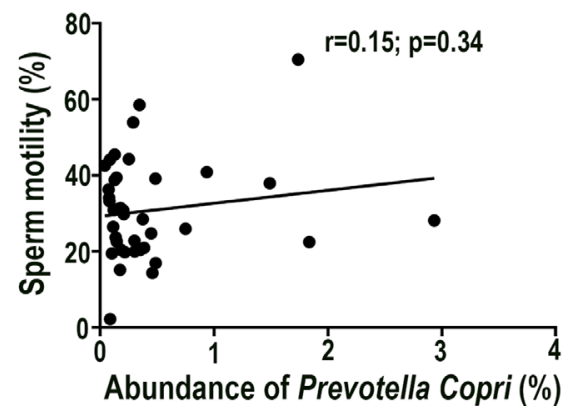

Figure 4 Correlation of gut Bacteroides and Prevotella collected from clinical sample with sperm motility and blood endotoxin. (A) The combined abundance of the genus Bacteroides-Prevotella collected from healthy donors and the patients with asthenozoospermia, oligozoospermia and teratozoospermia was negatively correlated with sperm motility, $n=60$ in total. (B) The abundance of Bacteroides in the gut of healthy donors and the patients with asthenozoospermia, oligozoospermia and teratozoospermia was positively correlated with blood endotoxin level, $n=60$ in total. LPS, lipopolysaccharide. (C) The correlation between the abundance of Prevotella copri and sperm motility in all 60 faecal samples. The dots in the red circle represent the abundance of $P$. copri $>15 \%$, whereas those in the black circle represent the abundance of $P$. copri $<3 \%$. (D) The correlation between the subpopulation with high abundance of $P$. copri $(>15 \%)$ and sperm motility $(n=20, p<0.05)$. (E) The correlation between the subpopulation with low abundance of $P$. copri $(<3 \%)$ and sperm motility $(\mathrm{n}=40)$.

The functional linkage between gut microbial dysbiosis and the decrease in sperm quality led us to investigate if clinical male infertility patients may have similar issues in gut microbes. Indeed, sequencing and bioinformatics analysis of the clinical faecal samples collected from some healthy donors and the patients with asthenozoospermia, oligozoospermia and teratozoospermia revealed that the combined abundance of BacteroidesPrevotella displayed a strong negative correlation with sperm motility (figure 4A). In contrast, the blood endotoxin levels of these clinical subjects showed a strong positive correlation with the abundance of Bacteroides (figure 4B). Thus, the potential linkage between the elevation of Bacteroides and Prevotella with the elevation of endotoxin and the defects in spermatogenesis and sperm motility may exist in both humans and mice.
Analysis of the clinical faecal samples further revealed that $P$. copri, a dominant species within Prevotella, could be an important species contributing to the spermatogenic defect. Interestingly, within all of the samples from our patients, the abundance of P. copri at the overall species level displayed a 'bipolar' trend at either $<3 \%$ or as high as $15 \%-80 \%$ (figure $4 \mathrm{C}$ ). When combining all the data together, the analysis showed a negative, and yet statistically not significant, association trend between sperm motility and the abundance of P. copri (figure 4C). However, after focusing on those patients' samples with the abundance of P. copri $>15 \%$, the results revealed a strong negative correlation $(n=20, r=-0.56, p=0.011$; figure 4D) that did not exist in those samples with the abundance of $P$. copri $<3 \%$ (figure 4E), suggesting that high abundance of $P$. copri or other unidentified 
species within Prevotella and Bacteroides could both play critical roles in regulating sperm production.

\section{HFD-induced dysbiosis of gut microbiota leads to infiltration of $T$ cells and macrophages in lamina propria of small intestine and epididymis inflammation}

The results described above demonstrated the elevation of blood endotoxin concentration and its strong association with some of the microbial community (Bacteroides, in particular) after transplanting the faecal microbes collected from the HFD-fed mice. Endotoxin has been known to regulate intestinal inflammation through a toll-like receptor 4 (TLR4) and myeloid differentiation factor 88 (MyD88)-dependent pathway. ${ }^{35}$ Real-time PCR assay found that the expression of TLR4 and MyD88 was both significantly elevated in the small intestinal regions (online supplementary figure $5 \mathrm{~A}$ ), whereas MyD88, but not TLR4, was increased in the colons (online supplementary figure $5 \mathrm{~B}$ ), suggesting that endotoxin primarily acted through small intestines by interacting with TLR4. Such observation led us to speculate that local chronic inflammation may also be provoked by endotoxin as a result of faecal transplantation. To this end, we first examined the inflammatory condition in small intestines of the recipient mice. H\&E staining displayed strong infiltration of lymphocytes in the lamina propria of the small intestine from the HFD-FMT mice (figure 5A). Immunohistochemistry and real-time PCR assays further revealed the presence of at least $\mathrm{T}$ cells (figure $5 \mathrm{~B}, \mathrm{C}$ ) and macrophages (figure 5D,E), which was indicative of a local inflammatory condition. The caput epididymis is a region where sperm undergoes maturation and acquires motile capacity. ${ }^{36}$ Elevation of endotoxin is known to be able to elicit immune responses in epididymis and damage sperm motility. ${ }^{38} 39$ Indeed, we found several pro-inflammatory cytokines, specifically interleukin (IL)-1 1 , C-X-C motif chemokine (CXCL)-10 and monocyte chemoattractant protein (MCP)-1 were significantly elevated in the epididymis of HFD-FMT mice (figure 5F). Taken together, our results suggest that increased endotoxin and at least local inflammation may have contributed to the impairment of sperm qualities.

\section{HFD-induced dysbiosis of gut microbiota altered testis gene expression}

The results described above have demonstrated the damage of spermatogenesis by the transplantation of HFD-induced gut microbiota. To further delineate the mechanisms underlying the impaired sperm production in the testis of HFD-FMT mice, we collected the whole testis samples for RNA-sequencing. The threshold of expression was set at FPKM $\geq 3$. Of those genes that met the requirement, 716 differentially expressed genes were identified with a greater than twofold expression change. Among them, 133 genes were upregulated and 583 genes were downregulated (figure 6A) in the samples derived from the HFD-FMT mice. Kyoto Encyclopedia of Genes and Genomes (KEGG) pathway analysis showed enrichment in a series of pathways, such as oxidative phosphorylation $\left(\mathrm{p}=0.42 \times 10^{-7}\right)$, protein export $(p=0.00025)$, steroid biosynthesis $(p=0.00053)$ and protein processing in endoplasmic reticulum $(p=0.0011)$ (figure 6B), all of which are critical to the process of spermatogenesis. In particular, a series of mitochondrial genes encoding different subunits of mitochondrial dehydrogenase complex I, including MT-ND1, MT-ND2, MT-ND4, MT-ND4L and MT-ND5 were significantly decreased (figure 6C,D).

RNA-sequencing also revealed significant reduction of those genes involved in the regulation of meiosis, particularly some of the structural maintenance of chromosomes (SMC) proteins (Smc1b, Smc3, Smc4, Smc5, Smc6) and synaptonemal complex proteins (Sycp1, Sycp2 and Sycp3) (figure 6C,E), indicating that the decreased expression of testicular Smc and Sycp genes induced by the gut microbial dysbiosis could have contributed to the drop in sperm production. In addition, we noted that other genes that play important roles in spermatogenesis were also affected in the testis of the HFD-FMT group. Accordingly, Crisp2 (ND-FMT $1440 \pm 84.26$ vs $870.6 \pm 54.38$ FPKM; $p<0.01)$ and Ggnbp2 (ND-FMT $164.90 \pm 19.43$ vs $77.39 \pm 4.62$ FPKM; $p<0.01$ ) were significantly decreased in the HFD-FMT group.

\section{DISCUSSION}

The present study has revealed that the dysbiosis of gut microbiota induced by HFD was one of the primary causes for the impaired sperm production and motility. Such phenotype is likely mediated by elevated blood endotoxin, epididymal inflammation and the dysregulation of testicular gene expressions. To the best of our knowledge, the findings in this study represent the first to establish a functional linkage between HFD-induced gut microbiota dysbiosis and impairment of male fertility.

Most of published gut microbial studies using the FMT procedure entailed antibiotic pretreatment. However, we chose not to do so primarily out of the concern that the antibiotic pretreatment could lead to some metabolic dysregulation (including glucose intolerance and even development of obesity), ${ }^{3233}$ which could potentially complicate the interpretation of gut microbes on spermatogenesis. In fact, even with the antibiotic pretreatment, the HFD-FMT mice still turned out lower sperm motility and sperm counts compared with ND-FMT mice (online supplementary figure 6), indicating that the effects of HFD gut microbiota on sperm production and quality were independent of antibiotic usage. It should also be noted that this study applied FMT procedure with the faecal microbes that is different from those from the mucosal samples. ${ }^{40}$ Such methodology may limit the colonisation of the transplanted microbes in the mucosa, which requires constant FMT every 2 days to maintain the functional influence. Despite such limitation, the transplanted HFD gut microbes still exerted a negative impact on the spermatogenesis of the recipient mice. The influence of mucosal microbiome on spermatogenesis should be clarified in the future.

Although it is well known that HFD feeding disrupts the balance of gut microbial ecosystem, our microbial transplantation manipulation further pinpointed the dramatic increase in the colonisation of Bacteroides and Prevotella at the genus level as the likely contributing microbes to the disruption of spermatogenesis and sperm motility. Interestingly, the population increase of Bacteroides in the HFD-FMT mice mirrors similar increase in the HFD-fed mice, indicative of a successful colonisation of Bacteroides in the transplanted recipient gut. Of particular interest to this study, blood endotoxin reached even endotoxaemia level in the HFD-FMT mice. HFD feeding is already known to generate elevated blood endotoxin. ${ }^{41}$ This may have been mediated by gut microbiota dysbiosis since a study has shown that HFD-induced population increase of Bacteroides-Prevotella ${ }^{42}$ is positively associated with the elevation of circulating endotoxin. These results bear strong clinical significance because our analysis of male infertile patients with asthenozoospermia/oligozoospermia/teratozoospermia issues revealed very similar strong negative correlation between the combined abundance of Bacteroides and Prevotella with sperm motility and a strong positive association between the abundance of Bacteroides with patients' blood endotoxin concentrations. Furthermore, in these patients, we identified a strong negative correlation between sperm motility and high 

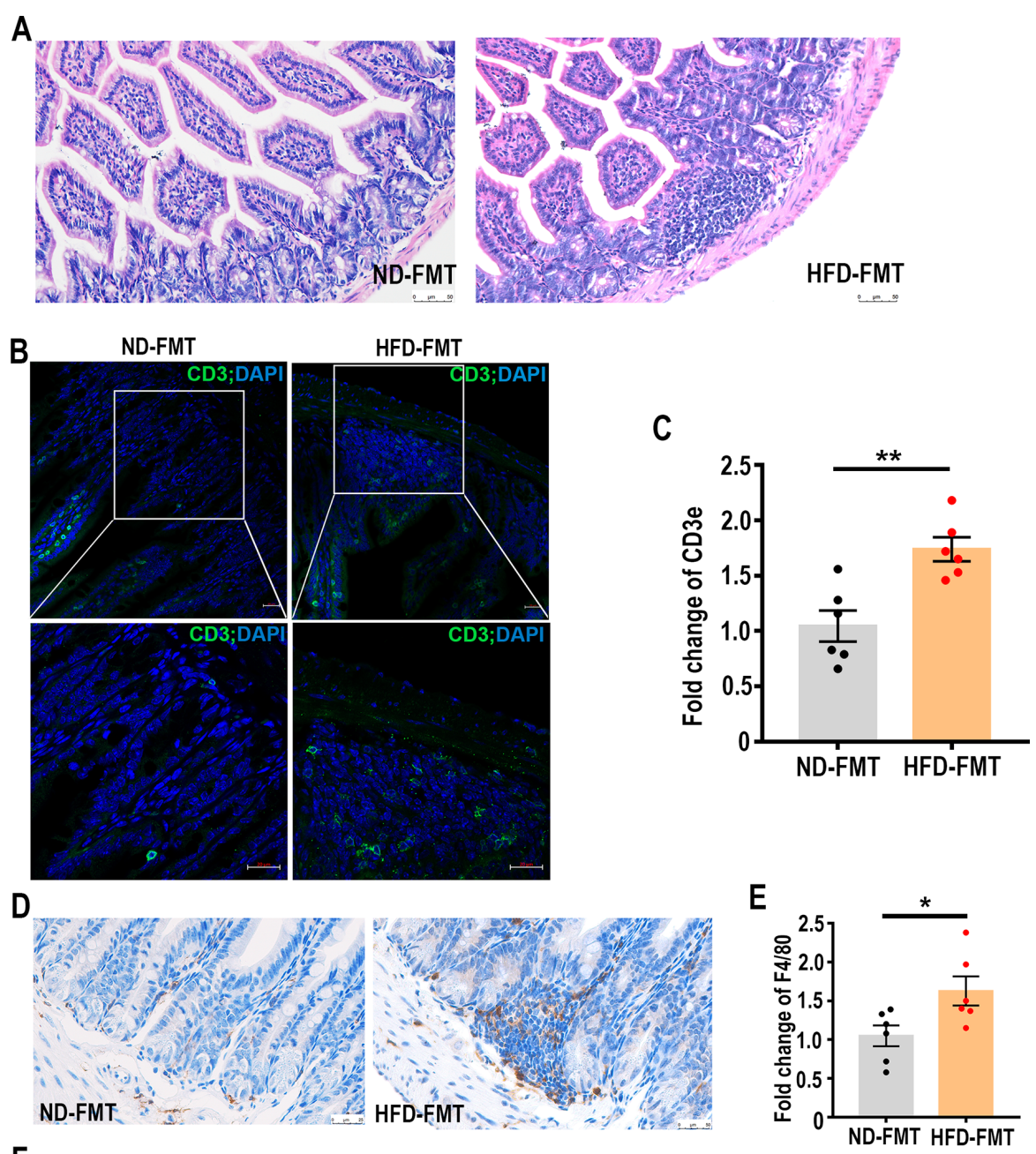

F

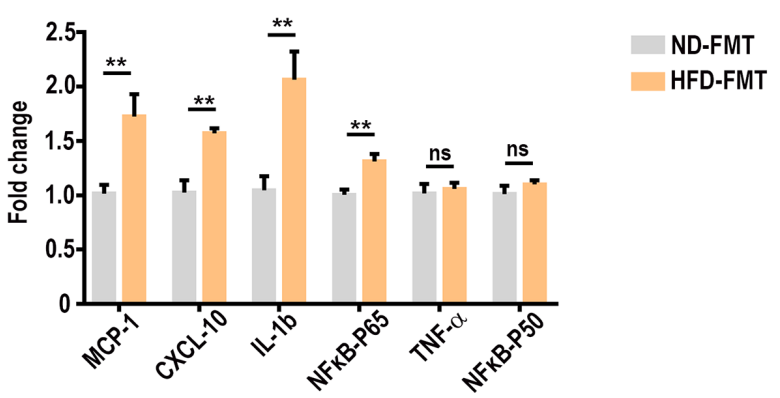

Figure 5 High-fat diet (HFD)-induced dysbiosis of gut microbiota leads to intestinal $\mathrm{CD}^{+} \mathrm{T}$ cell and macrophage aggregation and epididymal inflammation. (A) Histological examination of FMT-treated intestines. The sections were stained with H\&E. Scale bar=50 $\mu \mathrm{m}$. (B) Immunofluorescent analysis of CD3 (green) was performed in the small intestine samples of the microbe-transplanted mice. Nuclei were stained with DAPI (blue). The lower panels were enlarged from the upper panels. Scale bar $=20 \mu \mathrm{m}$. (C) The mRNA levels of CD3e in the small intestines of the microbe-transplanted mice were determined by real-time PCR assays. (D) Immunohistochemistry determination of macrophage infiltration in the small intestines of microbe-transplanted mice. Scale bar $=50 \mu \mathrm{m}$. (E) The mRNA levels of a macrophage marker, F4/80, in the small intestines of the microbe-transplanted mice were determined by real-time PCR assays. (F) The mRNA levels of pro-inflammation cytokines and NFkB-p65 in the caput epididymis of the microbe-transplanted mice were determined by real-time $P C R$ assays, $n=6$ for each group. ns, no significant difference. ${ }^{*} p<0.05$, ${ }^{* *} p<0.01$. $C X C L$, C-X-Cmotif chemokine; IL, interleukin; MCP, monocyte chemoattractant protein; NFKB, nuclear factor kappa B; TNF, tumour necrosis factor.

abundance of $P$. copri ( $>15 \%$ in overall species). P. copri, a dominant species within Prevotella genus, has been reported to be able to induce insulin resistance ${ }^{43}$ and to be more prevalent in the gut of patients with rheumatoid arthritis than in the healthy individuals. ${ }^{44}$ Our study adds a potential dimension of $P$. copri abundance influencing spermatogenesis. Of course, even the impact of $P$. copri on sperm production should be put in the context of HFD-induced gut ecosystem changes where other microbial species might also interact dynamically with $P$. copri. Thus, HFD-induced imbalance of gut microbial population, particularly the disturbance in Bacteroides and Prevotella (with P. copri acting as one of the potential 'culprits'), likely contributed to the spermatogenic defect and the increase of blood endotoxin.

The imbalance of HFD-induced gut microbiota can elicit host immune response ${ }^{22} 45$ that includes chronic inflammation. ${ }^{46}$ In 
A

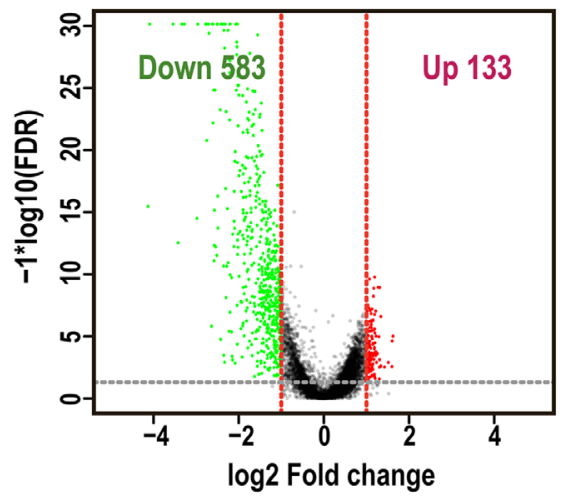

B
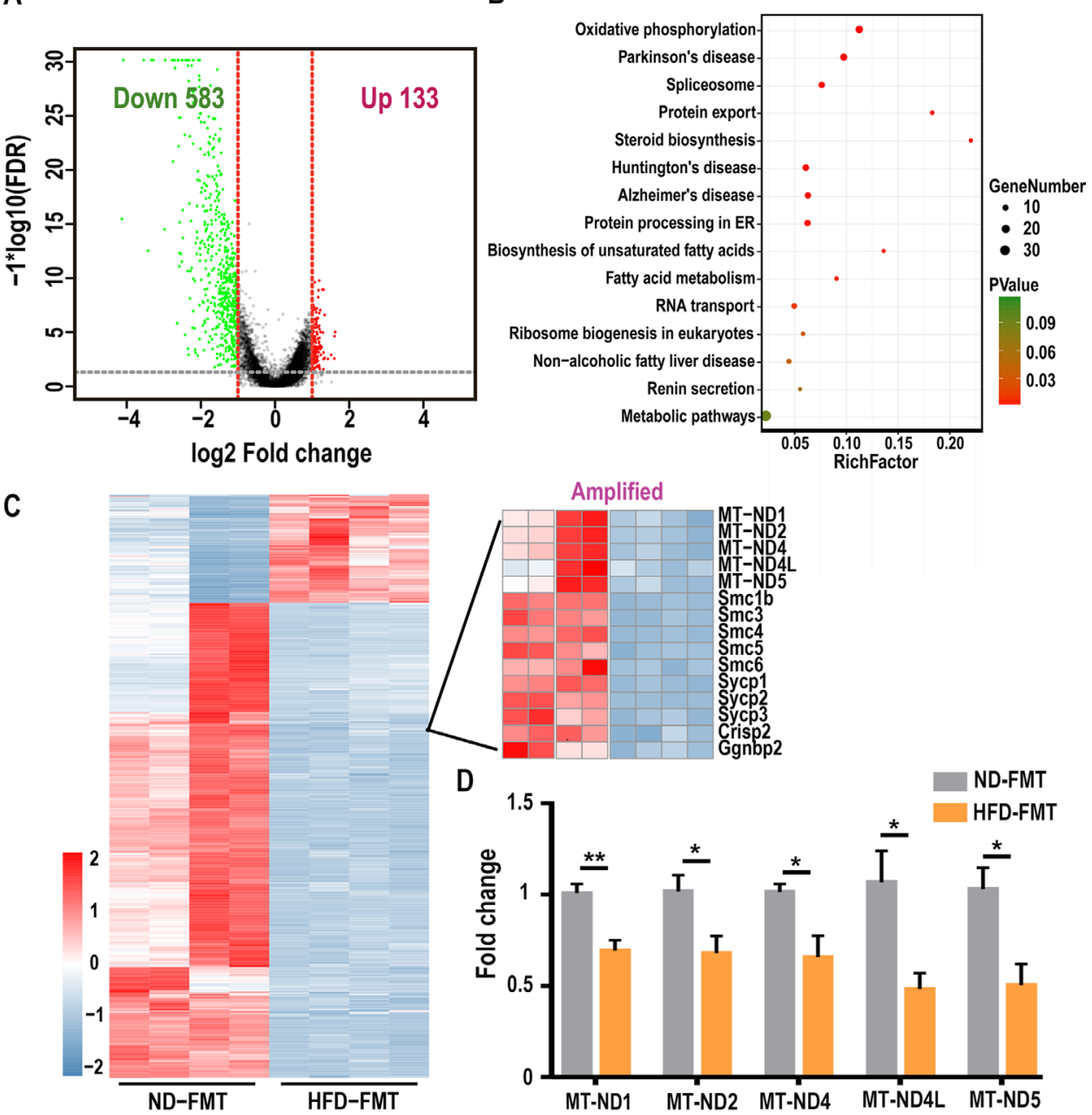

RichFactor

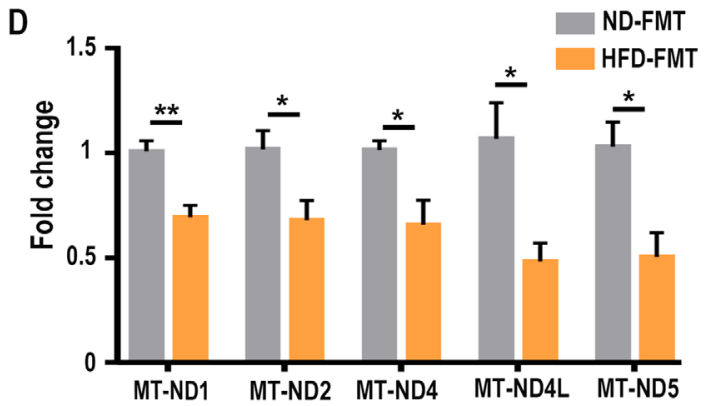

E

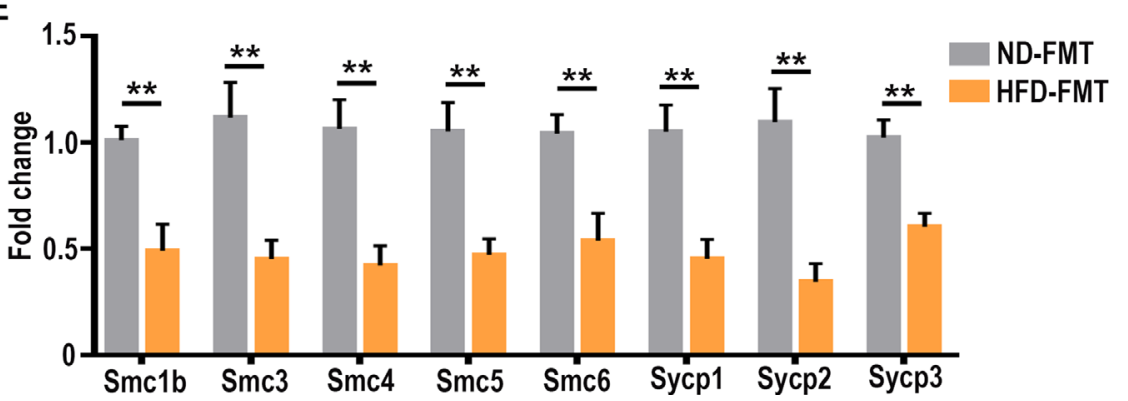

Figure 6 Alterations of gene expression in HFD-FMT treated testis. (A) Volcano plot showing the changes of testis genes (fold change $\geq 2$ ) in the HFD-FMT mice compared with the ND-FMT mice. Five hundred eighty-three genes were found to be downregulated (green dots) and 133 genes upregulated (red dots). The red lines indicate the log 2 fold change at 1 and $-1, n=4$ for each group. (B) Kyoto Encyclopedia of Genes and Genomes pathway enrichment analysis of differentially expressed genes, $n=4$ for each group. (C) Heat map of differentially expressed genes in ND-FMT and HFD-FMT treated testis, $n=4$ for each group. (D) Real-time PCR confirmations for the mitochondrial genes, $n=5$ for the ND-FMT group and $n=6$ for the HFD-FMT group. (E) The mRNA levels of meiosis relative genes in the testis were significantly downregulated in the HFD-FMT mice, detected by realtime PCR, $n=6$ for each group. ${ }^{*} p<0.05,{ }^{*} p<0.01$.

the mice transplanted with HFD-induced faecal microbes, we observed strong infiltration/aggregation of $\mathrm{T}$ cells and macrophages in the lamina propria of the small intestine, which by inference indicated a strong local inflammatory condition. Whether such localised intestinal inflammatory condition contributed to the observed spermatogenic defect remains to be defined. On the other hand, the caput epididymal inflammation, as reflected by the increased expression of IL-1 $\beta$, CXCL-10, MCP-1 and NF- $\mathrm{Bp} 65$, could be a likely 'culprit' responsible for the reduced sperm counts and motility in the HFD-FMT mice. Multiple studies have demonstrated that epididymal inflammation condition negatively affects sperm quality. ${ }^{38} 3947-52$ Global

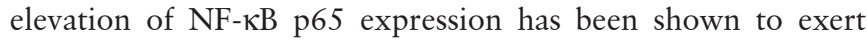
anti-inflammatory and antiobesity effects by increasing energy expenditure. ${ }^{53}$ However, we did not see any difference in energy expenditure between the two FMT-treated mice. This might be due to the fact that the elevation of NF- $\kappa \mathrm{B}$ p 65 expression was restricted to only certain organs/tissues such as the epididymis. 
Previous studies have well established the causal relationship between the endotoxaemia/epididymal inflammation and low sperm motility/spermatogenic dysfunction in both animal models and humans. ${ }^{38} 39$ 47-52 Intraperitoneal administration of lipopolysaccharides (LPS) could elevate the expression of pro-inflammatory cytokines, such as IL-1 $\beta$, IL- 6 and CXCLi2 in the epididymis and testis of roosters ${ }^{48}$ and increase the level of IL-18 in mouse testes. ${ }^{49}$ In addition, LPS injection could also elevate seminal plasma IL-1 $\beta$, TNF- $\alpha$, IL-6 levels and lead to low sperm motility in rats. ${ }^{50}$ Importantly, incubation of human spermatozoa with LPS could cause reduction in sperm motility. ${ }^{51}$ Metabolic endotoxaemia in human obesity is strongly linked with impaired sperm DNA integrity. ${ }^{52}$ These reports are consistent with our hypothesis that the endotoxaemia and epididymitis induced by faecal microbial transplantation led to the overt phenotypes in spermatogenesis. Thus, a structurally unbalanced architecture of gut microbiota, which was transmitted from the HFD mice, can exert a detrimental effect to host sperm motility via localised inflammation at the epididymis and systemic elevation of endotoxin.

Part of the defects in spermatogenesis as a result of transplantation of HFD-induced microbes happened at the level of disruption of testicular gene expression. In particular, we noted significant reduction in the expression of SMC serial genes (encoding cohesin, condensin and SMC5/6 complex proteins), and Sycp serial genes (encoding synaptonemal complex proteins). Members of these two gene families play pivotal roles in holding sister chromatids together or segregation, chromosome condensation, DNA double-strand breaks repairing as well as linking homologous chromosomes. ${ }^{54-59}$ In addition, the decrease of Ggnbp2 expression could have also negatively affected spermatogenesis since Ggnbp2 encodes a protein that is essential in DNA double-strand breaks repairing during meiotic progression of spermatocytes. ${ }^{60}$ The dysregulation of testicular gene expression could also lead to impaired sperm motility. Accordingly, RNA-sequencing and subsequent real-time PCR-validation revealed significant drop in the expression of some mitochondrial genes, including MT-ND1, MT-ND2, MT-ND4, MT-ND5 and MT-ND4L, all of which encode the core subunits of mitochondrial membrane respiratory chain, NADH dehydrogenase (complex I), ${ }^{61}$ that is essential for oxidative phosphorylation and sperm motility. ${ }^{62}$ Similarly, the diminution of Crisp2 expression, which is critical to regulate $\mathrm{Ca}^{2+}$ fluxes during sperm capacitation and sperm progressive motility, ${ }^{64} 65$ could have also contributed to the reduced sperm motility. Thus, the abnormal expression of those genes, which was caused by gut microbiota changes, may lead to the spermatogenic defect and low sperm motility.

Environmental and 'Westernised' dietary changes have long been thought as the important contributing factors to the protracted global decline of sperm concentrations in humans. Although 'Western-style' diet may disrupt male fertility partly through dysregulation of inflammation and glucose/lipid metabolism, this study offers an additional mechanistic dimension in linking the disrupted gut microbial ecosystem with impaired spermatogenesis and sperm motility. Owing to the complex interactions between the host and gut microbiota, additional work needs to be done to clarify the mechanisms underlying the impact of gut microbiota on male reproduction. The study presented herein highlights the possibility of treating male infertility, particularly those associated with metabolic syndromes, by restoring gut microbial ecosystem.

Contributors $X Z$ and ND performed the whole experiments. XYB, YJY, LLP, GMX and $X Z$ performed some experiments and analysed the data. SSL and XZ provided technical guidance. ND, YPM, BY and JJ collected and analysed human semen and faecal samples. HC, ND and AZZ wrote the manuscript. FHL and AZZ conceived and designed the experiments.
Funding This work was supported by grants from the National Key R\&D Program of China (2018YFA0800600 to AZZ); the National Natural Science Foundation of China (81630021 to AZZ); Key research and development program of Guangdong Province for "Innovative drug creation" (2019B020201015 to FHL); the Guangdong Innovative Research Team Program (2016ZT06Y432 to AZZ and FHL); the National Key Research and Development Program of China (2018YFC1004700 to BY); the National Natural Science Foundation of China (81701431 to JJ, 81871202 and 81671432 to HC, 81700690 to XYB); the National Program on Key Basic Research Project of China (2013CB945202 to AZZ and FHL); the China Postdoctoral Science Foundation (2018T110852 to XYB) and the Startup R\&D Funding of Guangdong University of Technology (50010102 to AZZ and FHL).

Competing interests None declared.

\section{Patient consent for publication Not required.}

Ethics approval This study was approved by Experimental Animal Ethics Committee of Guangdong Pharmaceutical University, Guangzhou, China. All clinical samples were collected under informed consent and approved by the Research Ethics Committee of Jinling Hospital.

Provenance and peer review Not commissioned; externally peer reviewed.

Data availability statement Data are available in a public, open-access repository. All data relevant to the study are included in the article or uploaded as supplementary information. All raw sequences have been deposited in the NCBI Sequence Read Archive (SRA) database (https://www.ncbi.nlm.nih.gov/). Accession number: SRP167405 (mRNA sequencing of testes in the FMT group), SRP221703 (16S-rDNA sequencing of clinical faecal samples), SRP168312 (16S-rDNA sequencing of FMT faeces). Data sets generated and analysed during this study are available from the corresponding author on reasonable request.

Open access This is an open access article distributed in accordance with the Creative Commons Attribution Non Commercial (CC BY-NC 4.0) license, which permits others to distribute, remix, adapt, build upon this work non-commercially, and license their derivative works on different terms, provided the original work is properly cited, appropriate credit is given, any changes made indicated, and the use is non-commercial. See: http://creativecommons.org/licenses/by-nc/4.0/.

\section{ORCID iDs}

Ning Ding http://orcid.org/0000-0002-1826-1749

Li Li Peng http://orcid.org/0000-0001-9818-8301

\section{REFERENCES}

1 Sharlip ID, Jarow JP, Belker AM, et al. Best practice policies for male infertility. Fertil Steril 2002;77:873-82.

2 Levine $\mathrm{H}$, Jørgensen $\mathrm{N}$, Martino-Andrade A, et al. Temporal trends in sperm count: a systematic review and meta-regression analysis. Hum Reprod Update 2017:23:646-59.

3 Virtanen HE, Jørgensen N, Toppari J. Semen quality in the 21st century. Nat Rev Urol 2017;14:120-30

4 Skakkebaek NE, Rajpert-De Meyts E, Buck Louis GM, et al. Male reproductive disorders and fertility trends: influences of environment and genetic susceptibility. Physiol Rev 2016;96:55-97.

5 Nordkap L, Jensen TK, Hansen Åse Marie, et al. Psychological stress and testicular function: a cross-sectional study of 1,215 Danish men. Fertil Steril 2016;105:174-87.

6 Li Y, Lin H, Li Y, et al. Association between socio-psycho-behavioral factors and male semen quality: systematic review and meta-analyses. Fertil Steril 2011;95:116-23.

7 Ramlau-Hansen CH, Toft G, Jensen MS, et al. Maternal alcohol consumption during pregnancy and semen quality in the male offspring: two decades of follow-up. Hum Reprod 2010;25:2340-5.

8 Hammoud AO, Wilde N, Gibson M, et al. Male obesity and alteration in sperm parameters. Fertil Steril 2008;90:2222-5.

9 Chavarro JE, Toth TL, Wright DL, et al. Body mass index in relation to semen quality, sperm DNA integrity, and serum reproductive hormone levels among men attending an infertility clinic. Fertil Steril 2010;93:2222-31.

10 Kort HI, Massey JB, Elsner CW, et al. Impact of body mass index values on sperm quantity and quality. J Androl 2006:27:450-2.

11 Bieniek JM, Kashanian JA, Deibert CM, et al. Influence of increasing body mass index on semen and reproductive hormonal parameters in a multi-institutional cohort of subfertile men. Fertil Steril 2016;106:1070-5.

12 Belloc S, Cohen-Bacrie M, Amar E, et al. High body mass index has a deleterious effect on semen parameters except morphology: results from a large cohort study. Fertil Steril 2014;102:1268-73.

13 Crean AJ, Senior AM. High-Fat diets reduce male reproductive success in animal models: a systematic review and meta-analysis. Obes Rev 2019:20:921-33.

14 Deshpande SS, Nemani H, Pothani S, et al. Genetically inherited obesity and highfat diet-induced obesity differentially alter spermatogenesis in adult male rats. Endocrinology 2019;160:220-34. 
15 Craig JR, Jenkins TG, Carrell DT, et al. Obesity, male infertility, and the sperm epigenome. Fertil Steril 2017;107:848-59.

16 Chen Q, Yan M, Cao Z, et al. Sperm tsRNAs contribute to intergenerational inheritance of an acquired metabolic disorder. Science 2016;351:397-400.

17 Wu GD, Chen J, Hoffmann C, et al. Linking long-term dietary patterns with gut microbial enterotypes. Science 2011;334:105-8.

18 Zhang $C$, Zhang $M$, Pang $X$, et al. Structural resilience of the gut microbiota in adult mice under high-fat dietary perturbations. Isme J 2012;6:1848-57.

19 David LA, Maurice CF, Carmody RN, et al. Diet rapidly and reproducibly alters the human gut microbiome. Nature 2014;505:559-63.

20 Turnbaugh PJ, Ley RE, Mahowald MA, et al. An obesity-associated gut microbiome with increased capacity for energy harvest. Nature 2006;444:1027-31.

21 Cani PD. Crosstalk between the gut microbiota and the endocannabinoid system: impact on the gut barrier function and the adipose tissue. Clin Microbiol Infect 2012;18 Suppl 4:50-3.

22 Tanoue T, Morita S, Plichta DR, et al. A defined commensal Consortium elicits CD8 T cells and anti-cancer immunity. Nature 2019;565:600-5.

23 Atarashi K, Tanoue T, Oshima K, et al. Treg induction by a rationally selected mixture of clostridia strains from the human microbiota. Nature 2013:500:232-6.

24 Gensollen T, lyer SS, Kasper DL, et al. How colonization by microbiota in early life shapes the immune system. Science 2016;352:539-44.

25 Leslie JL, Young VB. The rest of the story: the microbiome and gastrointestinal infections. Curr Opin Microbiol 2015;23:121-5.

26 Liu R, Hong J, Xu X, et al. Gut microbiome and serum metabolome alterations in obesity and after weight-loss intervention. Nat Med 2017;23:859-68.

27 Weingarden AR, Vaughn BP. Intestinal microbiota, fecal microbiota transplantation, and inflammatory bowel disease. Gut Microbes 2017;8:238-52.

28 Qin J, Li Y, Cai Z, et al. A metagenome-wide association study of gut microbiota in type 2 diabetes. Nature 2012;490:55-60.

29 Cooper DTG. WHO laboratory manual for examination and processing of human semen. 5th edn. World Health Organization, 2010.

30 Wang H, Cai Y, Shao Y, et al. Fish oil ameliorates high-fat diet induced male mouse reproductive dysfunction via modifying the rhythmic expression of testosterone synthesis related genes. Int J Mol Sci 2018;19:1325.

31 Palmer NO, Bakos HW, Owens JA, et al. Diet and exercise in an obese mouse fed a high-fat diet improve metabolic health and reverse perturbed sperm function. $A m$ J Physiol Endocrinol Metab 2012;302:E768-80.

32 Cox LM, Blaser MJ. Antibiotics in early life and obesity. Nat Rev Endocrinol 2015;11:182-90.

33 Lai Z-L, Tseng C-H, Ho HJ, et al. Fecal microbiota transplantation confers beneficial metabolic effects of diet and exercise on diet-induced obese mice. Sci Rep 2018;8:15625.

34 Grive KJ, Hu Y, Shu E, et al. Dynamic transcriptome profiles within spermatogonial and spermatocyte populations during postnatal testis maturation revealed by single-cell sequencing. PLoS Genet 2019;15:e1007810.

35 Płóciennikowska A, Hromada-Judycka A, Borzęcka K, et al. Co-Operation of TLR4 and raft proteins in LPS-induced pro-inflammatory signaling. Cell Mol Life Sci 2015;72:557-81.

36 Cornwall GA. New insights into epididymal biology and function. Hum Reprod Update 2009:15:213-27.

37 MacLean JA, Hayashi K, Turner TT, et al. The Rhox5 homeobox gene regulates the region-specific expression of its paralogs in the rodent epididymis. Biol Reprod 2012;86:189.

38 Wang F, Liu W, Jiang Q, et al. Lipopolysaccharide-Induced testicular dysfunction and epididymitis in mice: a critical role of tumor necrosis factor alphat. Biol Reprod 2019;100:849-61

39 Cao D, Li Y, Yang R, et al. Lipopolysaccharide-Induced epididymitis disrupts epididymal beta-defensin expression and inhibits sperm motility in rats. Biol Reprod 2010:83:1064-70.

40 Zmora N, Zilberman-Schapira G, Suez J, et al. Personalized gut mucosal colonization resistance to empiric probiotics is associated with unique host and microbiome features. Cell 2018;174:1388-405.
41 Cani PD, Amar J, Iglesias MA, et al. Metabolic endotoxemia initiates obesity and insulin resistance. Diabetes 2007:56:1761-72.

42 Cani PD, Bibiloni R, Knauf $C$, et al. Changes in gut microbiota control metabolic endotoxemia-induced inflammation in high-fat diet-induced obesity and diabetes in mice. Diabetes 2008; 57:1470-81.

43 Pedersen HK, Gudmundsdottir V, Nielsen HB, et al. Human gut microbes impact host serum metabolome and insulin sensitivity. Nature 2016;535:376-81.

44 Scher JU, Sczesnak A, Longman RS, et al. Expansion of intestinal Prevotella copri correlates with enhanced susceptibility to arthritis. Elife 2013;2:e01202.

45 Atarashi K, Tanoue T, Ando M, et al. Th17 cell induction by adhesion of microbes to intestinal epithelial cells. Cell 2015;163:367-80.

46 Wang J, Tang H, Zhang C, et al. Modulation of gut microbiota during probioticmediated attenuation of metabolic syndrome in high fat diet-fed mice. Isme J 2015;9:1-15.

47 Hedger MP. Toll-like receptors and signalling in spermatogenesis and testicular responses to inflammation-a perspective. J Reprod Immunol 2011;88:130-41.

48 Zhang M, Nii T, Isobe N, et al. Expression of Toll-like receptors and effects of lipopolysaccharide on the expression of proinflammatory cytokines and chemokine in the testis and epididymis of roosters. Poult Sci 2012;91:1997-2003.

49 Abu Elhija M, Lunenfeld E, Huleihel M. Lps increases the expression levels of IL-18, ice and IL-18 R in mouse testes. Am J Reprod Immunol 2008;60:361-71.

50 Collodel G, Moretti E, Brecchia G, et al. Cytokines release and oxidative status in semen samples from rabbits treated with bacterial lipopolysaccharide. Theriogenology 2015:83:1233-40.

51 Sahnoun S, Sellami A, Chakroun N, et al. Human sperm Toll-like receptor 4 (TLR4) mediates acrosome reaction, oxidative stress markers, and sperm parameters in response to bacterial lipopolysaccharide in infertile men. J Assist Reprod Genet 2017;34:1067-77.

52 Pearce KL, Hill A, Tremellen KP. Obesity related metabolic endotoxemia is associated with oxidative stress and impaired sperm DNA integrity. Basic Clin Androl 2019;29.

53 Tang T, Zhang J, Yin J, et al. Uncoupling of inflammation and insulin resistance by NF-kappaB in transgenic mice through elevated energy expenditure. J Biol Chem 2010;285:4637-44.

54 Lee J. Roles of cohesin and condensin in chromosome dynamics during mammalian meiosis. J Reprod Dev 2013:59:431-6.

55 Verver DE, van Pelt AMM, Repping S, et al. Role for rodent Smc6 in pericentromeric heterochromatin domains during spermatogonial differentiation and meiosis. Cell Death Dis 2013:4:e749.

56 Mehta GD, Rizvi SMA, Ghosh SK. Cohesin: a guardian of genome integrity. Biochim Biophys Acta 2012;1823:1324-42

57 Verver DE, Langedijk NSM, Jordan PW, et al. The SMC5/6 complex is involved in crucial processes during human spermatogenesis. Biol Reprod 2014;91:22.

58 de Vries FAT, de Boer E, van den Bosch M, et al. Mouse Sycp1 functions in synaptonemal complex assembly, meiotic recombination, and $X Y$ body formation. Genes Dev 2005; 19:1376-89.

59 Yuan L, Liu JG, Zhao J, et al. The murine SCP3 gene is required for synaptonemal complex assembly, chromosome synapsis, and male fertility. Mol Cell 2000;5:73-83.

60 Guo K, He Y, Liu L, et al. Ablation of Ggnbp2 impairs meiotic DNA double-strand break repair during spermatogenesis in mice. J Cell Mol Med 2018;22:4863-74.

61 Vinothkumar KR, Zhu J, Hirst J. Architecture of mammalian respiratory complex I. Nature 2014;515:80-4

62 DiMauro S, Schon EA. Mitochondrial respiratory-chain diseases. N Engl J Med 2003:348:2656-68.

63 Chai R-R, Chen G-W, Shi H-J, et al. Prohibitin involvement in the generation of mitochondrial superoxide at complex I in human sperm. J Cell Mol Med 2017;21:121-9.

64 Gibbs GM, Scanlon MJ, Swarbrick J, et al. The cysteine-rich secretory protein domain of Tpx-1 is related to ion channel toxins and regulates ryanodine receptor $\mathrm{Ca} 2+$ signaling. J Biol Chem 2006:281:4156-63.

65 Zhou J-H, Zhou Q-Z, Lyu X-M, et al. The expression of cysteine-rich secretory protein 2 (CRISP2) and its specific regulator miR-27b in the spermatozoa of patients with asthenozoospermia. Biol Reprod 2015;92:28. 